\title{
CHARACTERIZING PUISEUX MONOID DOMAINS WITH BOUNDED FACTORIZATION
}

\section{RYAN GIPSON}

Department of Mathematics

Campbellsville University

Campbellsville, KY 42718

USA

e-mail: rgipson@campbellsville.edu

\begin{abstract}
We consider general factorization properties of particular classes of integral domains. More specifically, we investigate the bounded factorization property of the monoid domain $F[X ; M]$, where $M$ is an additive submonoid of the nonnegative rational numbers, a ring of polynomial-like expressions where exponents come from $M$. We accomplish this task by examining sufficient and necessary factorization properties of the associated monoid $M$ and, in particular, its irreducibles. We divide our work into two sections: we first consider those bounded factorization domains with the finite factorization property, and, finally, we characterize all bounded factorization monoid domains by precisely determining the necessary factorization properties of its associated monoid.
\end{abstract}

2020 Mathematics Subject Classification: Primary 13F15, 13A05; Secondary 13G05, $20 \mathrm{M} 25$. Keywords and phrases: atomic domain, bounded factorization domain, monoid domain $F[X ; M]$.

Received January 8, 2021

(C) 2021 Scientific Advances Publishers 


\section{Introduction}

In 1986, Gilmer proposed a generic question, denoted $\left(Q_{E}\right)$, with his summary of analyses of semigroup rings in [7]. Here, $E$ is some ringtheoretic property and $Q$ is the question, "Under what conditions on $S$, a semigroup, and $R$, a commutative ring, does the semigroup ring $R[X ; S]$ satisfy $E$ ?" In [6] and [7], Gilmer provided some solutions to $\left(Q_{E}\right)$ for several ring-theoretic properties. One particularly interesting example, with respect to the discussions of this paper, is Gilmer's discovery of sufficient and necessary conditions for when a semigroup ring satisfies the unique factorization property.

More recently, several authors have continued along in similar investigations, choosing to analyze questions of this type, where $E$ is a more general factorization property, as did the authors in [8]. More specifically, the authors considered a special class of semigroup rings called monoid domains, denoted $F[X ; M]$, where $M$ is a subset of the additive monoid of nonnegative rationals, commonly referred to as Puiseux monoids. Interestingly, it was discovered in their paper that the factorization properties of these domains were a consequence of the generators (more specifically, the irreducibles) of the associated commutative monoid $M$. Thus, it became clear that investigations of factorization properties of $F[X ; M]$ must include an analysis of the elements in, and operations of, $M$. Indeed, current research corroborates this claim in relating the atomic property (respectively, half-factorial property) in the monoid $M$ to the atomic property (respectively, halffactorial property) in the associated domain as evidenced by [4] and [11], respectively. Interestingly, in the latter, the author showed that halffactorial Puiseux monoid domains are, in fact, unique factorization domains, since $F[X ; M]$ is half-factorial if and only if $M$ is cyclic. This leads one to wonder if, when $M \subseteq\left(\mathbb{Q}_{+},+\right)$, one factorization property of $F[X ; M]$ always necessitates a stronger factorization analogue in the monoid. 
We answer this question in the negative and provide both sufficient and necessary conditions of monoid domains with more general factorization properties, and we do so by analyzing the irreducibles of the associated monoid. To this end, we will first provide necessary preliminary notions and definitions in Section 2. We will then divide our work into two sections, first characterizing monoid domains with the finite factorization property (shown in [1] to be a subclass of bounded factorization domains) and then those with the more general bounded factorization property. In Sections 3 and 4, we characterize the respective domains according to analogous factorization properties in the associated monoids. In Section 5, we summarize our work by placing our results in the literature, providing some concluding remarks and observations along with some questions to guide further research.

\section{Preliminary Notions and Definitions}

We will be investigating properties of a special class of commutative rings called monoid rings. A monoid, written additively, is a nonempty set together with a binary associative operation + such that there is an identity element 0 . We say that a monoid is commutative if for every $x, y \in M, x+y=y+x$. We say that a set $A \subseteq M$ generates $M$, and write $\langle A\rangle=M$, if every element of $M$ can be written in the following way:

$$
\left\{k_{1} a_{1}+k_{2} a_{2}+\cdots+k_{n} a_{n} \mid n \geq 1, k_{i} \in \mathbb{N}, \text { and } a_{i} \in A\right\} .
$$

If $M$ can be generated by a finite set $A$, we say that $M$ is finitely generated; otherwise, we say that $M$ is infinitely generated. In this paper, we will always assume that $M \subseteq\left(\mathbb{Q}_{+},+\right)$, the monoid of nonnegative rationals. 
Given a commutative monoid, $M$, there is an associated ring $R$ of polynomial-like expressions, denoted $R=F[X ; M]$, where $F$ is a field and $X$ is the variable. The elements of $R$ are polynomials of the form

$$
f(X)=a_{0} X^{\alpha_{0}}+a_{1} X^{\alpha_{1}}+\cdots+a_{n} X^{\alpha_{n}}
$$

where $n \geq 0, a_{0}, \ldots, a_{n} \in F$, and $\alpha_{0}, \ldots, \alpha_{n} \in M$. If we assume $\alpha_{0}<\cdots$ $<\alpha_{n}$, then we say the degree of $f$, denoted $\operatorname{deg}(f)$, is $\alpha_{n}$. The set of units of $R$ is precisely the set of elements from the field $F$, i.e., the zero-degree polynomials of the ring. We call the ring $R$ a monoid ring. (It can be shown that $R$ is, in fact, an integral domain; hence, throughout this paper we will refer to $R$ as a monoid domain.)

We say that two monoids $M, M^{\prime} \subseteq\left(\mathbb{Q}_{+},+\right)$are isomorphic if there exists a map $\mu: M \rightarrow M^{\prime}$ such that the following hold: (1) $\mu(\alpha+\beta)=\mu(\alpha)+\mu(\beta)$ for all $\alpha, \beta \in M$ and (2) $\mu\left(0_{M}\right)=0_{M^{\prime}}$. We call $\mu$ a monoid isomorphism, and in [8] it is shown that every monoid isomorphism of submonoids of $\left(\mathbb{Q}_{+},+\right)$takes the form $\mu_{\tau}: M \rightarrow \tau M$, where $\tau \in \mathbb{Q}_{+} \backslash\{0\}$. For every monoid isomorphism $\mu$, there is an associated ring isomorphism $\phi_{\mu}: F[X ; M] \rightarrow F[X ; \mu(M)]$ defined by

$$
\phi_{\mu}\left(a_{0} X^{\alpha_{0}}+\cdots+a_{n} X^{\alpha_{n}}\right)=a_{0} X^{\mu\left(\alpha_{0}\right)}+\cdots+a_{n} X^{\mu\left(\alpha_{n}\right)} .
$$

We will investigate sufficient and necessary properties of $M$ such that $F[X ; M]$ has the following atomic factorization properties: finite factorization and bounded factorization. A nonzero element $u \in R$ is a unit if it is invertible with respect to multiplication. We say that a nonzero element $x \in R$ divides (or is a factor of) an element $z$ in the ring if there exists an element $y \in R$, such that $x y=z$. Two nonzero elements $x, y$ are said to be associates if we may write $x=u y$, where $u$ is a unit in $R$; we write $x \sim y$. A nonzero element $x \in R$ is irreducible, 
and called an atom, if whenever it is written as a product of two factors, say $x=y z, y$ or $z$ is a unit in the ring. If $x$ is not irreducible, we call it reducible.

We say that an integral domain $R$ has the atomic property, and we call it an atomic domain, if every nonzero, nonunit element $x \in R$ admits a factorization into a finite number of atoms. We say that $R$ has the bounded factorization property and call it a bounded factorization domain (or BFD) if it has the atomic property and if for every nonzero, nonunit element $x \in R$, there is an associated integer $n \geq 1$ such that for every factorization into irreducibles we have, say $x=p_{1} p_{2} \cdots p_{k}, k \leq n$. Moreover, we say that $R$, a $\mathrm{BFD}$, has the finite factorization property, and we call it a finite factorization domain (or FFD) if every nonzero, nonunit element $x \in R$ has finitely many essentially unique factorizations into irreducibles. (By essentially unique factorization into atoms, we mean the factorization is unique up to associates and order of factors.)

In our analysis of factorization properties of $F[X ; M]$, we will give particular consideration to the atoms of, and, furthermore, the factorization properties of the monoid $M$. In the case of additive monoids, we contextualize factorization with respect to addition rather than multiplication as demonstrated by Gilmer in [6]. Therefore, given a nonempty Puiseux monoid $M$, we may define the notions divides, unit, associates, reducible, and irreducible (or atom), analogous to similar notions in the domain. In addition, we say that two factorizations of $\alpha \in M$ into atoms are essentially equivalent if whenever $\alpha=\gamma_{1}+\gamma_{2}+$ $\cdots+\gamma_{k}$ and $\alpha=\delta_{1}+\delta_{2}+\cdots+\delta_{l}, k=l$ and for every $i \in\{1,2, \ldots, k\}$, there exists $j \in\{1,2, \ldots, l\}$, so that $\gamma_{i} \sim \delta_{j}$. Otherwise, we say the factorizations are essentially unique. It should be noted that it has been shown in [8] that a monoid domain whose associated monoid is not generated by atoms is not atomic. Thus, we may restrict our analysis to those domains where $M$ is finitely generated or $M$ is infinitely 
generated by atoms. In proving that every finitely generated submonoid of $\left(\mathbb{Q}_{+},+\right)$is generated by atoms, we first note that in [6] Gilmer showed that every numerical monoid, i.e., a submonoid of $(\mathbb{N},+)$, is generated by a finite set of natural numbers. Moreover, in [12], Gotti proved that every finitely generated Puiseux monoid is isomorphic to a numerical semigroup. Therefore, we must only show that every nonzero submonoid of $(\mathbb{N},+)$ is generated by atoms.

Proposition 2.1. Every submonoid $N \neq 0$ of $(\mathbb{N},+)$ is generated by atoms of $N$.

Proof. It follows from [6, Proposition 2.4] that $N$ is generated by a set, say $N=\left\langle a_{1}^{*}, a_{2}^{*}, \ldots, a_{k}^{*}\right\rangle$, where $k$ is minimum with respect to cardinality. We claim $a_{i}^{*}$ is an essential generator for $1 \leq i \leq k$. Indeed, suppose for some $j \in\{1,2, \ldots, k\}, a_{j}^{*}=x+y$, for nonzero elements $x, y \in M$. We then write $a_{j}^{*}=a_{x_{1}}+a_{x_{2}}+\cdots+a_{x_{l}}+a_{y_{1}}+a_{y_{2}}+\cdots+a_{y^{\prime}}$, where $\quad x=a_{x_{1}}+a_{x_{2}}+\cdots+a_{x_{l}}, y=a_{y_{1}}+a_{y_{2}}+\cdots+a_{y_{l}^{\prime}}$, and $a_{x_{1}}$, $a_{x_{2}}, \ldots, a_{x_{l}}, a_{y_{1}}, a_{y_{2}}, a_{y^{\prime}} \in\left\{a_{1}^{*}, a_{2}^{*}, \ldots, a_{k}^{*}\right\}$. Thus, $N=\left\langle a_{1}^{*}, a_{2}^{*}, \ldots, a_{j-1}^{*}\right.$, $\left.a_{j+1}^{*}, \ldots, a_{k}^{*}\right\rangle$ and, hence, $\left\{a_{1}^{*}, a_{2}^{*}, \ldots, a_{k}^{*}\right\}$ is not minimum in cardinality, a contradiction.

Unlike the finitely generated case, infinitely generated monoids are not necessarily generated by atoms. Indeed, $\left(\mathbb{Q}_{+},+\right)$is an example. However, in Example 4.10 of Section 4, we introduce a useful example of an infinitely generated submonoid of the nonnegative rationals, generated by atoms, namely the monoid generated by all fractions $1 / p$, where $p$ is a prime number.

In several of our proofs, we will make use of the following definition and lemmas without referencing them explicitly. (We will omit the proofs of the lemmas since they are straight forward.) 
Definition 2.2. We say that the element $\frac{m}{n} \in M$ is in reduced form if $\operatorname{gcd}(m, n)=1$.

Lemma 2.3. Let $\frac{m_{1}}{n_{1}}, \frac{m_{2}}{n_{2}} \in M$ be two fractions in reduced form. Then $\frac{m_{1}}{n_{1}}=\frac{m_{2}}{n_{2}}$ if and only if $m_{1}=m_{2}$ and $n_{1}=n_{2}$.

Lemma 2.4. Let $a X^{\alpha}$ be an element of $F[X ; M]$, where $M \subseteq\left(\mathbb{Q}_{+},+\right)$. If $f(X)$ divides $a X^{\alpha}$ in $F[X ; M]$, then $f(X)=b X^{\beta}$, and $\gamma=\alpha-\beta$ is an element of $M$.

Finally, we will describe a factorization process of a nonzero, nonunit element $f \in F[X ; M]$ into irreducibles. Suppose $f$ is reducible. We then factor $f$ into two nonzero, nonunit factors $f=f_{0} \cdot f_{1}$; if both $f_{0}, f_{1}$ are irreducible, then we stop the factorization process with one step. If either $f_{0}, f_{1}$ is reducible, after relabelling, we factor $f_{1}$ into two nonzero, nonunit factors and we have $f=f_{0} \cdot f_{1,0} \cdot f_{1,1}$. If each $f_{0}, f_{1,0}, f_{1,1}$ factor is irreducible, then we stop the factorization process after two steps. If any of the factors $f_{0}, f_{1,0}, f_{1,1}$ are reducible, after relabelling, we factor $f_{1,1}$ into two nonzero, nonunit factors and we have $f=f_{0} \cdot f_{1,0} \cdot f_{1,1,0}$. $f_{1,1,1}$. If each $f_{0}, f_{1,0}, f_{1,1,0}, f_{1,1,1}$ factor is irreducible, then we stop the factorization process after three steps. If not, after relabelling, we factor $f_{1,1,1}$ into two nonzero, nonunit factors. This process continues until each factor of $f$ is irreducible. If the factorization process terminates after a finite number of steps, we call it a finite factorization process of $f$; otherwise, we call it an infinite factorization process of $f$. (Making the appropriate changes, one may also describe a finite factorization process and infinite factorization process of nonzero elements in additive monoids, as demonstrated in [15], which we demonstrate to be quite useful in quickly ascertaining whether $M$ has the bounded factorization property.) 
With these notions, we will now investigate the bounded fractorization property of the monoid domain $F[X ; M]$ by investigating factorization properties of its associated monoid. Any other notion may be found in [13] or [16].

\section{When $F[X ; M]$ is an FFD}

We will begin our investigation into the finite factorization property of $F[X ; M]$ by defining its analogue in $M$ in the following way: every nonzero, nonunit element $\alpha \in M$ admits finitely many essentially unique factorizations into atoms of $M$. From this definition, one may deduce that $\alpha \in M \backslash\{0\}$ has finitely many atom factors, and, therefore, $\alpha \in M \backslash\{0\}$ has finitely many divisors. With these notions, we show how finite factorization in the monoid affects the factorizations of certain polynomials in $F[X ; M]$.

Lemma 3.1. Let $b X^{\alpha}$ be an element of $F[X ; M]$. Then $b X^{\alpha}$ is an atom if and only if $\alpha$ is an atom of $M$.

Proof. This follows as an instance of the more general case proved in [5], Lemma 3.1.

Lemma 3.2. Let $M \subseteq\left(\mathbb{Q}_{+},+\right)$be a monoid and $F[X ; M]$ be a monoid domain. Then $\alpha \in M \backslash\{0\}$ admits two essentially unique factorizations into atoms in the monoid if and only if the polynomial $X^{\alpha} \in F[X ; M]$ admits two essentially unique factorizations in the monoid domain.

Proof. Both implications follow immediately from Lemma 3.1.

Lemma 3.3. Let $M \subseteq\left(\mathbb{Q}_{+},+\right)$be a monoid and $F[X ; M]$ be a monoid domain. When $M$ is an FFM, the polynomial $X^{\alpha} \in F[X ; M]$, where $\alpha \neq 0$, has finitely many essentially unique factorizations into atoms in the domain. 
Proof. Follows from the definition of FFM and Lemma 3.2

Lemma 3.4. Let $M \subseteq\left(\mathbb{Q}_{+},+\right)$be a monoid and $F[X ; M]$ be a monoid domain. If $M$ is an FFM, then the polynomial $X^{\alpha} \in F[X ; M]$ has finitely many factors.

Proof. Follows from Lemma 3.2 and the definition of FFM.

Proposition 3.5. Let $M \subseteq\left(\mathbb{Q}_{+},+\right)$be a monoid and $F[X ; M]$ be a monoid domain. If $M$ is an FFM, then $F[X ; M]$ is a BFD.

Proof. By Lemma 3.4, the leading monomial of some polynomial $f(X)=X^{\alpha_{n}}+a_{n-1} X^{\alpha_{n-1}}+\cdots+a_{1} X^{\alpha_{1}}$ has finitely many irreducible factors. Let $k \in \mathbb{N}$ be so that every factorization of $X^{\alpha_{n}}$ into irreducibles, say $X^{\alpha_{n}}=X^{\gamma_{1}} \cdot X^{\gamma_{2}} \ldots X^{\gamma_{l}}$, where $\gamma_{1}, \gamma_{2}, \ldots, \gamma_{l} \in M$ are atoms, $l \leq k$. Consider the following factorization process of $f(X)$ in the domain:

$$
\begin{aligned}
f(X) & =f_{0}(X) \cdot f_{1}(X) \\
& =f_{0}(X) \cdot f_{1,0}(X) \cdot f_{1,1}(X) \\
& =f_{0}(X) \cdot f_{1,0}(X) \cdot f_{1,1,0}(X) \cdot f_{1,1,1}(X)
\end{aligned}
$$

Since at each step $i$ of the factorization process $X^{\alpha_{n}}$ is equal to the product of the $i+1$ leading terms of the $i+1$ polynomial factors, the factorization process must end in no more than $k-1$ steps, and, thus, $f(X)$ can be factored into no more than $k$ factors.

Having shown that monoid domains with associated finite factorization monoids are bounded factorization domains, we now provide a sufficient condition for the existence of finite factorization monoid domains. 
Theorem 3.6. Let $M \subseteq\left(\mathbb{Q}_{+},+\right)$be a monoid and $F[X ; M]$ be a monoid domain. Then $F[X ; M]$ is a finite factorization domain if $M$ is a finite factorization monoid and there are no convergent sequences of elements, with finitely many equal terms, from $M$.

Proof. $(\Rightarrow)$ Associated with every factorization of $\alpha \in M \backslash\{0\}$ into atoms, say $\alpha=\gamma_{1}+\gamma_{2}+\cdots+\gamma_{n}$, is the corresponding factorization of the polynomial $X^{\alpha} \in F[X ; M]$. That is

$$
X^{\alpha}=X^{\gamma_{1}} X^{\gamma_{2}} \cdots X^{\gamma_{n}}
$$

where $X^{\gamma_{i}}$ is an atom in the domain follows from Lemma 3.1. If, then, $\alpha$ admits infinitely many essentially unique factorizations in $M$ into atoms, then $X^{\alpha}$ does the same, by Lemma 3.2, in $F[X ; M]$, a contradiction.

$(\Leftarrow)$ Take $f(X)$ to be a polynomial in $F[X ; M]$, defined as

$$
f(X)=X^{\alpha_{n}}+a_{n-1} X^{\alpha_{n-1}}+\cdots+a_{1} X^{\alpha_{1}}+a_{0} X^{\alpha_{0}},
$$

where $n \geq 0$ and $\alpha_{n}>\alpha_{n-1}>\cdots>\alpha_{1}>\alpha_{0}$. If $n=0$, Lemma 3.3 says $f(X)$ has finitely many essentially unique factorizations into atoms. Suppose $n \geq 1$, then, and also that $f(X)$ admits infinitely many essentially unique factorizations into atoms, say

$$
\begin{aligned}
f(X) & =f_{1,1}(X) \cdot f_{1,2}(X) \cdots f_{1, k_{1}}(X) \\
& =f_{2,1}(X) \cdot f_{2,2}(X) \cdots f_{2, k_{2}}(X) \\
& =f_{3,1}(X) \cdot f_{3,2}(X) \cdots f_{3, k_{3}}(X)
\end{aligned}
$$

where each equation may be written

$$
\begin{aligned}
f(X)= & X^{\beta_{\alpha n, 1}+\cdots+\beta_{\alpha n, k_{n}}}+\cdots+a_{1} X^{\beta_{\alpha_{1}, 1}+\cdots+\beta_{\alpha_{1}, k_{1}}} \\
& +a_{0} X^{\beta_{\alpha_{0}, 1}+\cdots+\beta_{\alpha_{0}, k_{0}}}+g_{i, 1} X^{\gamma_{i, 1}}+\cdots+g_{i, l_{i}} X^{\gamma_{i, l_{i}}} .
\end{aligned}
$$


By Lemma 3.4, $X^{\alpha_{i}}$ has finitely many divisors in the domain; hence, for fixed elements $\beta_{\alpha_{r}, s} \in M$, there are infinitely many equations of the form above and each can be written as:

$$
f(X)=f(X)+g_{i, 1} X^{\gamma_{i, 1}}+g_{i, 2} X^{\gamma_{i, 2}}+\cdots+g_{i, l_{i}} X^{\gamma_{i, l_{i}}}
$$

and we see, clearly, that $g_{i, 1} X^{\gamma_{i, 1}}+g_{i, 2} X^{\gamma_{i, 2}}+\cdots+g_{i, l_{i}} X^{\gamma_{i, l_{i}}}=0$. Now, for all $p \in\{0,1, \ldots, n\}$, define the sets: $E_{p}=\left\{\beta_{\alpha_{p}, q} \mid 1 \leq q \leq k_{p}\right\}$, and for all $i, G_{i}=\left\{\gamma_{i, j} \mid 1 \leq j \leq l_{i}\right\}$, and their respective unions

$$
\begin{aligned}
& E=\bigcup_{p=0}^{n} E_{p} \text { and } \\
& G=\bigcup G_{i} .
\end{aligned}
$$

Note, since $\left|E_{p}\right|<\infty$ for all $p,|E|<\infty$; thus, if $|G|<\infty$, their union $|E \cup G|<\infty$. In this case, we define the set of denominators of $E \cup G$ by $D_{E \cup G}=\left\{b \in \mathbb{N} \mid \frac{a}{b} \in E \cup G\right.$ is in reduced form $\}$, and we compute $\tau=\operatorname{lcm}\left(D_{E \cup G}\right)$. We consider, then, the monoid isomorphism $\mu_{\tau}: M \rightarrow \tau M \quad$ and its associated ring isomorphism $\phi_{\mu_{\tau}}: F[X ; M] \rightarrow F[X ; \tau M]$, where we have $\phi_{\mu_{\tau}}(f(X))$ and infinitely many essentially unique factorizations of $\phi_{\mu_{\tau}}(f(X))$ into atoms, represented by Equations (1) and (2), in $F[X]$. This is impossible; therefore, $f(X)$ admits only finitely many essentially unique factorizations in $F[X ; M]$. 
Now, suppose $|G|=\infty$. Since $g_{i, 1} X^{\gamma_{i}, 1}+g_{i, 2} X^{\gamma_{i}, 2}+\cdots+g_{i, l_{i}} X^{\gamma_{i, l_{i}}}=0$, for all $i, \alpha_{0}<\gamma_{i, j}<\alpha_{n}$ for all $i, j$. Hence, there are infinitely many elements in $M$ contained within the real interval $\left[\alpha_{0}, \alpha_{n}\right]$, and, thus, there exists a convergent sequence of elements from $M$.

As we conclude our investigations of finite factorization monoid domains, we will turn our attention briefly to finite factorization monoids. Observe, our hypothesis for Theorem 3.6 contains two conditions on the monoid. However, the condition that there are no convergent sequences of elements from $M$ can be shown to imply that $M$ is, in fact, an FFM. Indeed, we show this sufficiency condition below, and we begin with a well-known fact.

Lemma 3.7. Let $n$ be a positive integer. Then the number of distinct positive integer sums equalling $n$ is bounded by $2^{n-1}$.

Lemma 3.8. Let $N \subseteq\left(\mathbb{N}_{+},+\right)$be a nonzero monoid. Then for any element, say $a \in N \backslash\{0\}$, the number of distinct positive integer sums equalling $n$ is bounded by $2^{n-1}$.

Proposition 3.9. Let $M=\left\langle\frac{m_{1}}{n_{1}}, \frac{m_{2}}{n_{2}}, \cdots, \frac{m_{k}}{n_{k}}\right\rangle$, where $k \geq 1$ and each $\frac{m_{i}}{n_{i}}$ is in reduced form, be a submonoid of $\left(\mathbb{Q}_{+},+\right)$. Then for any element, $\frac{m}{n} \in M \backslash\{0\}$, the number of distinct sums of nonzero rationals in $M$, equalling $\frac{m}{n}$, is at most $2^{n-1}$.

Proof. Follows immediately from [12, Proposition 3.2] and Lemma 3.8. 
Lemma 3.10. Let $E \subseteq \mathbb{Q}_{+}$so that $m \in E$ is minimum and $M \in E$ is maximum. If $E$ contains infinitely many elements, then there exists a sequence of elements from $E$, say $s_{n}=\left\{a_{1}, a_{2}, a_{3}, \ldots\right\}$, with finitely many equal terms, which converges in the interval $[m, M] \subset \mathbb{R}$.

Proposition 3.11. Let $M \subseteq\left(\mathbb{Q}_{+},+\right)$be an infinitely generated bounded factorization monoid. If $\alpha \in M$ has infinitely many essentially unique factorizations into atoms, then there exists a sequence of atoms, with finitely many equal terms, from $M$ which converges, not necessarily in $M$.

Proof. Since $M$ is a BFM, there exists some integer $N>1$ so that when $\alpha$ is factored into atoms in the monoid, say $\alpha=\gamma_{1}+\gamma_{2}+\cdots+\gamma_{k}$, $k \leq N$. Thus, $\alpha$ has infinitely many unique factorizations into atoms of length $k^{\prime} \leq N$. That is, we have

$$
\begin{aligned}
\alpha & =\gamma_{1_{1}}+\gamma_{1_{2}}+\cdots+\gamma_{1_{k^{\prime}}} \\
& =\gamma_{2_{1}}+\gamma_{2_{2}}+\cdots+\gamma_{2_{k^{\prime}}} \\
& =\gamma_{3_{1}}+\gamma_{3_{2}}+\cdots+\gamma_{{k^{\prime}}^{\prime}} \\
& \vdots \\
& =\gamma_{n_{1}}+\gamma_{n_{2}}+\cdots+\gamma_{n_{k^{\prime}}}
\end{aligned}
$$

Note, here, that although the atoms in the factorizations above are not necessarily distinct, there must be infinitely many distinct atoms present in them. Otherwise, each equation, $\alpha=\gamma_{i_{1}}+\gamma_{i_{2}}+\cdots+\gamma_{i_{k^{\prime}}}$, could be written as

$$
\alpha=n_{1} \gamma_{1}^{*}+n_{2} \gamma_{2}^{*}+\cdots+n_{l} \gamma_{l}^{*}
$$


where $n_{1}, n_{2}, \ldots, n_{l}$ are nonnegative integers and $\gamma_{1}^{*}, \gamma_{2}^{*}, \ldots, \gamma_{l}^{*}$ are atoms, and according to Proposition 3.9, there are only finitely many unique sums represented by Equation (3). Therefore, we have

$$
\begin{aligned}
\alpha & =\widehat{\gamma_{1}}+\beta_{1} \\
& =\widehat{\gamma_{2}}+\beta_{2} \\
& =\widehat{\gamma_{3}}+\beta_{3} \\
& \vdots \\
& =\widehat{\gamma_{n}}+\beta_{n}
\end{aligned}
$$

where $\widehat{\gamma_{1}}, \widehat{\gamma_{2}}, \ldots$ are distinct atoms and $\beta_{1}, \beta_{2}, \ldots$ are elements from the monoid. Knowing that, for all $i, 0<\widehat{\gamma_{i}} \leq \alpha$, we have from Lemma 3.10, that there exists a convergent sequence of atoms.

Corollary 3.12. Let $M \subseteq\left(\mathbb{Q}_{+},+\right)$be an infinitely generated bounded factorization monoid. If $\alpha \in M$ has infinitely many essentially unique factorizations into atoms, then there exists an infinite number of distinct sequences of elements, each having finitely many equal terms, from $M$ which converge, not necessarily in $M$.

Here, it is natural to ask if there exists a finite factorization monoid from which a sequence of elements may be constructed, having finitely many equal terms, which converges. We answer this question in the positive, with an example below, thus showing that Theorem 3.6 does not necessarily provide necessary conditions for when $F[X ; M]$ is an FFD. Afterward, we provide an important question to guide further investigations. 
Example 3.13. The monoid $M=\left\langle\frac{p_{1}}{q_{1}}, \frac{p_{2}}{q_{2}}, \ldots, \frac{p_{i}}{q_{i}}, \ldots\right\rangle$, where each $p_{i}, q_{j}$ is a prime number, $p_{i}<q_{i}, p_{i}<p_{i+1}, q_{i}<q_{i+1}, \frac{p_{i}}{q_{i}}>\frac{p_{i+1}}{q_{i+1}}$, and $p_{i} \neq q_{j}$ for all $i, j$, is a FFM.

Proof. It can be shown that $M$ is atomic and that each $\frac{p_{i}}{q_{i}}$ is an atom. Let $\frac{m}{n} \in M$ be a nonzero, reducible element. By Proposition 3.9, it suffices to show that $\frac{m}{n}$ has finitely many atomic divisors. We begin by showing that when $\frac{m}{n}$ is expressed as a linear combination of atoms, each addend in reduced form, then the denominator of each addend must divide $n$. Indeed, write

$$
\frac{m}{n}=\frac{n_{1} p_{1}}{q_{1}}+\frac{n_{2} p_{2}}{q_{2}}+\cdots+\frac{n_{k} p_{k}}{q_{k}}
$$

where $k>1, n_{1}, \ldots, n_{k}$ are nonnegative integers, and each addend is in reduced form. By combining the fractions on the right-hand side of the equation, we get

$$
\frac{m}{n}=\frac{n_{1} p_{1} q_{2} q_{3} \cdots q_{k}+n_{2} p_{2} q_{1} q_{3} \cdots q_{k}+\cdots+n_{k} p_{k} q_{1} q_{2} \cdots q_{k-1}}{q_{1} \cdots q_{k}}
$$

and each side of Equation (4) is in reduced form; hence, $n=q_{1} \cdots q_{k}$. For every addend, then, $\frac{n_{i} p_{i}}{q_{i}}$ of $n$, where $q_{i}$ is greater than every prime divisor of $n, q_{i}$ must divide $n_{i}$. Hence, $\frac{n_{i} p_{i}}{q_{i}} \geq p_{i}$, and for some positive integer $N$, if $i>N, \frac{n_{i} p_{i}}{q_{i}} \nmid \frac{m}{n}$, when $n_{i} \neq 0$. Therefore, $\frac{m}{n}$ has only finitely many irreducible factors in the monoid. 
Question 3.14. What conditions on the elements of $M \subseteq\left(\mathbb{Q}_{+},+\right)$are necessary for $M$ to be an FFM?

\section{When $F[X ; M]$ is a BFD}

In our work to characterize those Puiseux domains exhibiting the bounded factorization property, we will find it helpful to divide our work into two cases: (1) the case where $M \subseteq\left(\mathbb{Q}_{0},+\right)$ is finitely generated and (2) the case where $M \subseteq\left(\mathbb{Q}_{0},+\right)$ is infinitely generated. The former is relatively simple, since it is easy to prove that $F[X ; M]$, where $N$ is a submonoid of $\left(\mathbb{N}_{0},+\right)$, is, in fact, a BFD, and from [12, Proposition 3.2], we have that every finitely generated Puiseux monoid $M$ is isomorphic to a submonoid from $\left(\mathbb{N}_{0},+\right)$. It is important to note this may also be easily deduced from [10, Theorem 3.1], where Gotti showed that all finitely generated Puiseux monoids have the bounded factorization property, and general factorization properties of particular classes of integral domains.

In directing our attention, then, to the case where $M$ is infinitely generated, we utilize notions of factorization processes in both the domain and the monoid, and we are able to recharacterize some factorization properties of integral domains (and, similarly, their monoid analogues). For example, to show that an integral domain is an atomic domain, it is sufficient to show that every nonzero, nonunit element admits a finite factorization process. Moreover, one sees that every atomic domain has this property. From [8], we have that when $M$ cannot be generated by atoms, $F[X ; M]$ is nonatomic, i.e., it is not a BFD. Unlike the finite case, however, it is well known that not every monoid domain, whose associated monoid is generated by infinitely many atoms, is a BFD. Indeed, we provide two examples below, only one of which has the bounded factorization property; we will delay verification of these claims until the end of the section. 
Example 4.1. Let $M=\left\langle\frac{1}{2}, \frac{1}{3}, \frac{1}{5}, \frac{1}{7}, \frac{1}{11}, \ldots, \frac{1}{p}, \ldots\right\rangle$, where $p$ is a prime number. Then $M$ is generated by atoms and $F[X ; M]$ is not a bounded factorization domain.

Example 4.2. Let $M=\left\langle\frac{1}{2}, \frac{2}{3}, \frac{3}{4}, \frac{4}{5}, \ldots, \frac{n}{n+1}, \ldots\right\rangle$, where $n \geq 1$ is an integer. Then $M$ is generated by atoms and $F[X ; M]$ is a bounded factorization domain.

We will consider, now, the bounded factorization property of monoids. We say the monoid $(M,+)$ has the bounded factorization property, and call it a bounded factorization monoid (or a BFM), if it is atomic, and if for every nonunit element $\alpha$ in the monoid, there is a positive integer $N$, such that if $\alpha=\gamma_{1}+\gamma_{2}+\cdots+\gamma_{k}$, where $\gamma_{1}, \gamma_{2}, \ldots, \gamma_{k}$ are atoms of the monoid, then $k \leq N$. Equivalently, we may define BFMs in terms of factorization processes in the monoid.

Proposition 4.3. A Puiseux monoid $M$ is a bounded factorization monoid if and only if for every nonzero element $\alpha \in M$, there exists a positive integer $N$ so that every factorization process of $\alpha$ in the monoid terminates in $\leq N$ steps.

Proof. $(\Leftarrow)$ First, we observe that since every factorization process terminates, every nonzero element of $M$ can be expressed as a sum of atoms; thus, $M$ is atomic. Next, we note that at step $k$ of any factorization process of $\alpha$, there are $k+1$ factors. If every factorization process of $\alpha$ in $M$ terminates in at most $N$ steps, any factorization of $\alpha$ into atoms in $M$ has at most $N+1$ factors. Therefore, $M$ has the bounded factorization property. 
$(\Rightarrow)$ Suppose $M$ is a BFM, take $\alpha \in M$ to be nonzero, and consider an arbitrary factorization process of $\alpha$, say,

$$
\begin{aligned}
\alpha & =\alpha_{0}+\alpha_{1} \\
& =\alpha_{0}+\alpha_{1,0}+\alpha_{1,1} \\
& =\alpha_{0}+\alpha_{1,0}+\alpha_{1,1,0}+\alpha_{1,1,1}
\end{aligned}
$$

Since $M$ is atomic, the process must terminate at some step $k<N^{*}$, where every factorization of $\alpha$ into atoms can have at most $N^{*}$ factors; otherwise, we have a contradiction. Set $N=N^{*}$ and we are finished.

The utility of the factorization process extends into the domain $F[X ; M]$, as well. With it we are able to prove the following results of factorization in the ring by primarily considering factorization in the associated monoid. We omit the majority of the proofs, since Proposition 4.3 is sufficient, as we will see in Theorem 4.9, to classify Puiseux domains with the bounded factorization property.

Lemma 4.4. Let $M \subseteq\left(\mathbb{Q}_{+},+\right)$be a bounded factorization monoid and let $\alpha \in M$ be nonzero. Then every factorization process of $\alpha$ is finite.

Lemma 4.5. Let $M \subseteq\left(\mathbb{Q}_{+},+\right)$be a bounded factorization monoid. Then every factorization process of the polynomial $X^{\alpha} \in F[X ; M]$ is finite.

Lemma 4.6. Let $M \subseteq\left(\mathbb{Q}_{+},+\right)$be a bounded factorization monoid. Then for every polynomial $X^{\alpha} \in F[X ; M]$, there is a positive integer $N$ such that when $X^{\alpha}$ is factored into atoms, say

$$
X^{\alpha}=X^{\gamma_{1}} \cdot X^{\gamma_{2}} \cdots X^{\gamma_{k}}
$$

it implies $k \leq N$. 
Proposition 4.7. Let $M \subseteq\left(\mathbb{Q}_{+},+\right)$be a bounded factorization monoid. Then $F[X ; M]$ is an atomic domain.

Proof. We will show that every factorization process of the domain is finite. Let $f(X)=X^{\beta_{1}}+b_{2} X^{\beta_{2}}+\cdots+b_{n} X^{\beta_{n}}$, where $n \geq 1$ and $\beta_{1}>\beta_{2}$ $>\cdots>\beta_{n}$, be a polynomial in $F[X ; M]$. By way of contradiction, suppose

$$
\begin{aligned}
f(X) & =f_{0}(X) \cdot f_{1}(X) \\
& =f_{0}(X) \cdot f_{1,0}(X) \cdot f_{1,1}(X) \\
& =f_{0}(X) \cdot f_{1,0}(X) \cdot f_{1,1,0}(X) \cdot f_{1,1,1}(X)
\end{aligned}
$$

Letting $X^{\alpha_{0}}, X^{\alpha_{1}}, X^{\alpha_{1,0}}, X^{\alpha_{1,1,0}}, X^{\alpha_{1,1,1}}, \ldots$ be the leading terms for each of the polynomials $f_{0}(X), f_{1}(X), f_{1,0}(X), f_{1,1,0}(X), f_{1,1,1}(X), \ldots$, respectively, we have then the following factorization process of $X^{\beta_{1}}$ :

$$
\begin{aligned}
X^{\beta_{1}} & =X^{\alpha_{0}} \cdot X^{\alpha_{1}} \\
& =X^{\alpha_{0}} \cdot X^{\alpha_{1,0}} \cdot X^{\alpha_{1,1}} \\
& =X^{\alpha_{0}} \cdot X^{\alpha_{1,0}} \cdot X^{\alpha_{1,1,0}} \cdot X^{\alpha_{1,1,1}}
\end{aligned}
$$

a contradiction, by Lemma 4.5. Hence, $F[X ; M]$ is atomic.

Proposition 4.8. Let $M \subseteq\left(\mathbb{Q}_{+},+\right)$be a bounded factorization monoid. Then $F[X ; M]$ is a bounded factorization domain. 
Proposition 4.8 also is a corollary to a strong result submitted previously by Anderson and Juett in [2, Theorem 13].

In summarizing our analysis of the bounded factorization property in $F[X ; M]$, we present our theorem.

Theorem 4.9. Let $M \subseteq\left(\mathbb{Q}_{+},+\right)$. Then, the following are equivalent:

(1) for every nonzero element $\alpha \in M$, there exists a positive integer $N$ so that every factorization process of $\alpha$ in the monoid terminates in $\leq N$ steps;

(2) $M$ is a bounded factorization monoid;

(3) $F[X ; M]$ is a bounded factorization domain.

Proof. (1) $\Leftrightarrow(2)$ Follows from Proposition 4.3.

(2) $\Leftrightarrow(3)$ The authors in [2, Theorem 13] proved that for any integral domain $R$ and $M$, a reduced torsion-free monoid, $R[X ; M]$ is a BFD if and only if $R$ is a BFD and $M$ is a BFM. Our statements hold since $F$ is a field and every Puiseux monoid is both reduced and torsion-free.

Our theorem proves especially useful in quickly determining whether a given monoid domain has the bounded factorization property, since one may consider an arbitrary factorization process of any nonzero element of $M$. We provide some examples below. In each case, we have omitted those portions of the proof showing that the monoid is atomic, preferring to demonstrate the utility of the factorization process instead.

Example 4.10. Let $M=\left\langle\frac{1}{2}, \frac{1}{3}, \frac{1}{5}, \frac{1}{7}, \frac{1}{11}, \ldots, \frac{1}{p}, \ldots\right\rangle$, where $p$ is a prime number. Then $M$ is generated by atoms and $F[X ; M]$ is not a bounded factorization domain. 
Proof. It is not difficult to show that each element $\frac{1}{p} \in M$, where $p$ is a prime number, is an atom in the monoid. To show that $F[X ; M]$ is not a BFD, one simply needs to consider the element $1 \in M$ and the factorization process

$$
\begin{aligned}
1 & =\frac{1}{p}+\frac{p-1}{p} \\
& =\frac{1}{p}+\frac{1}{p}+\frac{p-2}{p} \\
& \vdots \\
& =\underbrace{\frac{1}{p}+\frac{1}{p}+\cdots+\frac{1}{p}}_{p \text {-times }} .
\end{aligned}
$$

Thus, 1 admits factorization processes of whose set of lengths is unbounded, and $F[X ; M]$ is not a BFD.

Example 4.11. Let $M=\left\langle\frac{1}{2}, \frac{2}{3}, \frac{3}{4}, \frac{4}{5}, \ldots, \frac{n}{n+1}, \ldots\right\rangle$, where $n \geq 1$ is an integer. Then $M$ is generated by atoms and $F[X ; M]$ is a bounded factorization domain.

Proof. Suppose $\alpha$ is a nonzero element of the monoid and consider an arbitrary factorization process of $\alpha$. That is,

$$
\begin{aligned}
\alpha & =\alpha_{0}+\alpha_{1} \\
& =\alpha_{0}+\alpha_{1,0}+\alpha_{1,1} \\
& =\alpha_{0}+\alpha_{1,0}+\alpha_{1,1,0}+\alpha_{1,1,1}
\end{aligned}
$$


Note, at each step $k$ of the factorization process, the right-hand side of the equation is $\geq \frac{k+1}{2}$. Thus, we have the sequence

$$
\left\{a_{n}\right\}=\left\{1, \frac{3}{2}, 2, \frac{5}{2}, \ldots, \frac{k+1}{2}, \ldots\right\}
$$

where each $a_{k}$ is a lower bound for the right-hand side of the equation at each step $k$ of the factorization process. This sequence is unbounded; thus, there is an integer $N \geq 1$ such that the right-hand side of the equation is greater than $\alpha$ for all $k \geq N$. Hence, the factorization process must terminate prior to step $N$; therefore, it is bounded and $F[X ; M]$ is a BFD.

Example 4.12. Let $M=\{0\} \cup(1, \infty) \cap \mathbb{Q}$ and show that $F[X ; M]$ is a BFD.

Proof. Since $M \subseteq\left(\mathbb{Q}_{+},+\right)$and one may show that $M=\langle(1,2] \cap \mathbb{Q}\rangle$ and that every generator in the interval is an atom, we need only prove that it is a BFM. So, we consider a factorization process of a nonzero element of the monoid, say,

$$
\begin{aligned}
\alpha & =\alpha_{0}+\alpha_{1} \\
& =\alpha_{0}+\alpha_{1,0}+\alpha_{1,1} \\
& =\alpha_{0}+\alpha_{1,0}+\alpha_{1,1,0}+\alpha_{1,1,1}
\end{aligned}
$$

Note, at each step $k$, the sum on the right hand side is $>k+1$. Let $N$ be the minimum positive integer such that $N(k+1)>\alpha$. Then the factorization process must terminate before step $k=N$. Thus, we may apply our theorem and conclude that $F[X ; M]$ is a BFD. 


\section{Concluding Remarks}

With these results, we have succeeded in characterizing Puiseux monoid domains which have the bounded factorization property, according to factorization processes in the associated monoid. Our investigations have also echoed some natural questions concerning more general factorization properties of monoid domains, as well as, the associated monoid.

Question 5.1. What factorization properties of the monoid $M$ are necessary and sufficient to characterize all atomic monoid domains $F[X ; M]$ ?

Some of the work for Question 5.1 has been completed. Indeed, in [15], Kulosman characterized those domains which are ACCP and by doing so constructed a simple example for an atomic non-ACCP integral domain. Also, as noted in the introduction, recent work has been completed with regards to the atomic and half-factorial properties.

One recalls that Proposition 3.11 gives sufficient conditions for when the monoid $M \subseteq\left(\mathbb{Q}_{+},+\right)$has the finite factorization property. (Some sufficient conditions have also been presented for bounded factorization.) It would prove helpful, then, if one determined, precisely, those properties of $M$ which yield finite factorization in the associated domain, as well as, those properties of $M$ which necessitate the bounded factorization property in $M$. These improve the usefulness of Theorem 4.9 as illustrated in Example 4.12. This brings us to our final question.

Question 5.2. What properties of $M \subseteq\left(\mathbb{Q}_{+},+\right)$characterize precisely the factorization in the monoid?.

We acknowledge that some of the work on Question 5.2 has been completed. In [6], the author characterized GCD-monoids, unique factorization monoids et al. Also, in [10], the author has provided many sufficient conditions for several atomic factorization properties in submonoids of the nonnegative rationals. 


\section{Acknowledgement}

The authors would like to thank an anonymous referee for several useful suggestions and comments, improving the quality of this paper.

\section{References}

[1] D. D. Anderson, D. F. Anderson and M. Zafrullah, Factorization in integral domains, Journal of Pure and Applied Algebra 69(1) (1990), 1-19.

DOI: https://doi.org/10.1016/0022-4049(90)90074-R

[2] D. D. Anderson and J. R. Juett, Long length functions, Journal of Algebra 426 (2015), 327-343.

DOI: https://doi.org/10.1016/j.jalgebra.2014.12.016

[3] S. T. Chapman, F. Gotti and M. Gotti, A survey on the atomicity of Puiseux monoids,

Available on arXiv: https://arxiv.org/pdf/1908.09227.pdf

[4] J. Coykendall and F. Gotti, On the atomicity of monoid algebras, arXiv: 1906.11138 [math. AC].

[5] J. Coykendall and B. Mammenga, An embedding theorem, Journal of Algebra, 325(1) (2011), 177-185.

DOI: https://doi.org/10.1016/j.jalgebra.2010.08.026

[6] R. Gilmer, Commutative Semigroup Rings, The University of Chicago Press, Chicago, 1984.

[7] R. Gilmer, Property E in commutative monoid rings, group and semi-group rings (G. Karpilovsky, Editor), Elsevier Science Publishers, B. V. (North-Holland) (1986), 13-18.

[8] R. Gipson and H. Kulosman, Atomic and AP semigroup rings $F[X ; M]$, where $M$ is a submonoid of the additive monoid of nonnegative rational numbers, International Electronic Journal of Algebra 22(22) (2017), 133-146.

DOI: https://doi.org/10.24330/ieja.325939

[9] R. Gipson and H. Kulosman, For which additive submonoids $M$ of nonnegative rationals is $F[X ; M]$ AP?, preprint.

[10] F. Gotti, The elasticity of Puiseux monoids, arXiv:1703.04207 [math.AC].

[11] F. Gotti, Irreducibility and factorizations in monoid rings, arXiv:1905.07168 [math. AC].

[12] F. Gotti, On the atomic structure of Puiseux monoids, Journal of Algebra and its Applications 16(7) (2017); Article 1750126.

DOI: https://doi.org/10.1142/S0219498817501262 
[13] I. Kaplansky, Commutative Rings, Revised Edition, The University of Chicago Press, Chicago and London, 1974.

[14] H. Kim, Factorization in monoid domains, Communications in Algebra 29(5) (2001), 1853-1869.

DOI: https://doi.org/10.1081/AGB-100002153

[15] H. Kulosman, A new simple example of an atomic domain which is not ACCP, Advances in Algebra 12(1) (2019), 1-7.

[16] D. G. Northcott, Lessons on Rings, Modules and Multiplicities, Cambridge University Press, Cambridge, 1968.

DOI: https://doi.org/10.1017/CBO9780511565922 